\title{
Loading of Quartz Oscillator Plates
}

\author{
Leland T. Sogn and Philip A. Simpson
}

\begin{abstract}
The effects of physically loading the surface of two types of piezoelectric quartz oscillator plates have been experimentally investigated. Various amounts of Wood's metal were applied to different parts of the crystal surfaces. Thickness-shear-mode plates were benefited only when the metal was applied on or near the active central area of the plate, but $\mathrm{X}$-cut crystals employing an extensional mode of vibration responded favorably only when the metal was applied near the periphery of the plate.

In both types of oscillator plates loading increased the crystal unit $Q$ several fold. Activity-frequency characteristics over a temperature range $-60^{\circ}$ to $+90^{\circ} \mathrm{C}$ were greatly improved.
\end{abstract}

\section{Introduction}

Piezoelectric quartz crystal units are extensively employed as frequency controlling and filtering devices in electronic circuits because of their extremely constant equivalent electrical parameters and high Q. However, coupled with the fundamental vibrational mode there are secondary modes of vibration that, under conditions favorable to them, often become so active that they affect both the activity and frequency of the fundamental controlling mode as well as other critical parameters of the crystal equivalent circuit. Various methods, such as face contouring and edge dimensioning, have been used to minimize the effects of this coupling. Experiments recently concluded at the Bureau indicate that selective physical loading on the piezoelectric quartz-plate surface gives approximately equivalent results.

\section{Preliminary Experiments}

Observations made on 0.5 - by 0.6 -in. hand-finished quartz plates a few years ago yielded many interesting data that provided the basis for the present investigation. When the electrodes coupling the crystal to the oscillator circuit were placed on the thicker portion of a wedge-shaped plate, not only was the frequency lower but the activity was higher than when they were placed on the thinner portion of the plate. In order to investigate this phenomenon more eftectively, a small brass electrode $1 / 4 \mathrm{in}$. in diameter at the base was constructed. This electrode made it possible to couple much smaller portions of the quartz plate to the oscillator circuit and to study the effect of coupling to local irregularities in the crystal surface that resulted from the application of nonuniform finger pressure during grinding. As expected, it was possible to excite a large number of frequencies by coupling areas of different thickness to the oscillator circuit. The previously mentioned inverse frequency-activity relationship was apparent and was found to be very consistent as the electrode was moved about on the surface of the plate. It was noted that whenever a certain fre- quency was excited by coupling to different areas of the crystal plate the activity was the same. It was also noted that the lowest frequency was associated with the highest activity.

Similar studies were made later on machine-lapped, slightly convex quartz plates. Results were much the same, except that random distribution of frequencies was replaced by orderly progression as the electrode was moved from the center along a diagonal toward a corner of the plate. Areas to which the oscillator circuit could be coupled in order to excite a certain frequency were arranged in the form of concentric rings about the center, with the lowest frequencies near the center and the highest at the corners.

The frequency associated with the highest activity was not the lowest frequency obtained by coupling to the exact center of the plate, but was a slightly higher one excited by coupling to an area immediately surrounding the center. In a plate whose frequency increased $25 \mathrm{kc}$ between center and corner, the frequency with the highest activity was 4 or 5 kc higher than that of the center. It was this fact that suggested the hypothesis that for each crystal plate there is one frequency and electrode position that will give the highest activity, and that physical loading of the plate would be beneficial through causing it to oscillate nearer this optimum frequency.

\section{Detailed Experiments}

\subsection{Concave Crystals}

The first crystals loaded were a group of $10-\mathrm{Mc}$ plates, 0.6 by 0.5 in., with their centers approximately $0.00005 \mathrm{in}$ thinner than the corners. The activities (rectified oscillator grid current) of these plates unloaded varied between 0.0 and 0.2 ma over the range $-60^{\circ}$ to $+90^{\circ} \mathrm{C}$. The frequency curves also were very poor, showing many irregularities.

The plates were then etched 3 to $5 \mathrm{kc}$ and loaded back the same amount so as to maintain the same relationship between their fundamental and secondary modes. The load was Wood's metal arranged in a ring concentric with the center of the plate and 
approximately $1 / 4$ in. in diameter. It was applied with several light strokes rather than one heavy one.

The activity of these plates increased to $0.7 \mathrm{ma}$ or more, and subsequent temperature tests revealed that the frequency curves, as well as the activity curves, were much smoother.

In order to investigate further the effect of loading, several 10-Mc crystals were etched and loaded in approximately 1 -kc steps. The crystals showed immediate improvement and continued to improve until lowered 6 kc by loading, after which further loading resulted in a slight loss in activity (fig. 1). Moreover, the crystals, which in the unloaded condition worked intermittently, oscillated throughout the temperature range; the fluctuations decreased in number and in size (fig. 2 ).

\subsection{Flat Crystals}

AT-cut plates, 0.6 by 0.5 in., of a lower frequency also were prepared and showed a favorable response to loading. The plates, whose frequencies were around 3,700 and $5,500 \mathrm{kc}$, had a very slight convex contour, less than 0.00001 in. They were etched in 5-kc steps and loaded back to the original frequency. The activity began to increase at once, and best results were attained with a load reducing the frequency 35 to $45 \mathrm{kc}$ (fig. 3). Further loading resulted in a decrease in activity. Evidently the load had the same effect as contouring, that is, it created an area of low frequency in the center of the plate.

Several different patterns of loading were used. Best results were obtained when the major length of the load was parallel to the X-direction, although a circular pattern did give good results with concave crystals. Besides the circular pattern, an elliptical load, wide-band load, narrow-band load, and pinhead load were used (fig. 4). A narrow band applied parallel to the $\mathrm{Z}^{\prime}$-direction appeared to have a deleterious effect on the performance. The most favorable pattern appeared to be the elliptical one, although the wide-band load also improved the crystal to a large degree. The narrow-band load in the Xdirection produced only a slight improvement, which never approached that of the elliptical or wide band. In general, it appeared that a load was most beneficial when its shape conformed to that of the active central area of the oscillator plate.

Interesting results were obtained with a pinhead size load. Initial application of load decreased the frequency as expected. However, after the crystal fundamental had been reduced about 0.2 percent, the frequency suddenly jumped to many kilocycles above the fundamental of the unloaded crystal. Explorations with a small probe electrode revealed that the plate was inert along a line through the center parallel to the $\mathrm{X}$-axis, and that each half of the plate was oscillating. Lycopodium powder patterns further confirmed this.

Measurements were made on 0.6 - by 0.5 -in. ATcut plates with the $\mathrm{X}$-direction parallel to the long dimension. Their frequency was approximately
6,702 kc. Elliptical loads differing in length, but not width, were used. The plates were etched and then loaded back to their original frequency so as to maintain as closely as possible the original relationship between primary and secondary modes.

Associated with the general improvement of a crystal unit there is, as expected, an increase in its $Q$, or quality factor. A crystal unit may be represented as a tuned circuit as shown in figure 5, where $C_{1}$ is the equivalent series (motional) capacity, $R_{s}$ the series resistance, $L_{1}$ the equivalent series inductance, and $C_{t}$ the total effective capacity shunting the series resonant circuit. The $Q$ of this circuit may then be determined by the formula

$$
Q \approx \frac{1}{4 \pi \Delta f R_{s} C_{t}},
$$

where $\Delta f=f_{a}-f_{r}$, the difference between the antiresonance and resonance of the crystal unit.

The loading of the crystal plate affected $\Delta f$ and $R_{s}$ very much but produced, at most, a negligible change in $C_{t}$. Both $\Delta f$ and $R_{s}$ tended to decrease at first, resulting in a rise in $Q$. At some loads, dips in the $Q$ occurred, probably due to coupling between the fundamental and some other mode associated with the oscillator plate. However, the crystal unit $Q$ was still several times the original one.

When the frequency change produced by the load reached approximately 1 percent of the fundamental, $R_{s}$ began to increase, resulting in a drop in activity. However, the $Q$ continued upward because $\Delta f$ was still decreasing (fig. 6). Further loading caused the frequency to jump to a value several kilocycles above that of the unloaded plate (fig. 7). It appeared that the loaded area became so far off frequency that a thinner region farther from the center became active. In other words, this was just another case of the previously mentioned "pinhead jump."

The area of the load also had a definite effect on the $Q$. For a load one-third the length of the $\mathrm{X}$-dimension and 0.3 the width of the $\mathrm{Z}^{\prime}$, the $Q$ of the 6,702 -kc plate rose steeply to a high value and remained high until shifted $65 \mathrm{kc}$. Attempts to shift $70 \mathrm{ke}$ resulted in a frequency jump. For Joads longer in the $\mathrm{X}$-direction, the $Q$ rose less steeply, but heavier loads could be applied before the frequency jumped. In all cases, the $Q$ 's reached approximately the same maximum value. An increase in the width of a given load was accompanied by an increase in $\Delta f$, but its effect on $R_{s}$ was unpredictable.

\subsection{Off-Center Load}

In order to determine the effect of placing the load off-center, 12 BT-cut plates were prepared at approximately $4,930 \mathrm{kc}$. These crystals were 0.6 by 0.5 in., with the $\mathrm{X}$-axis parallel to the short dimension, and had a slight convexity. Areas $1 / 8,3 / 16$, or $1 / 4$ in. in diameter were then plated on both sides"with gold. 
They were located as shown in figure 8 . Contact was then made with the plating, so that the gold served as an electrode as well as a load. In order to map out the active area, a pencil point under moderate pressure was then moved from the edges toward the center until all oscillations ceased. The points in figure 8 show the position of the pencil when this occurred. The region within was considered the active area.

Two definite effects were noted. Although the electrodes were not over the center of the crystal and that region was therefore apparently outside the field created by the electrode, the central portion of the plate was still approximately in the center of the active area. However, the active area was shifted in that it also encompassed some of the region under the electrode and usually extended beyond it (fig. 8). The position of the load did not shift the region of activity in the central area very much, but did elongate the active area when placed off-center.

\subsection{X-cuts}

$\mathrm{X}$-cut oscillator plates have their best performance characteristics if their contour is concave rather than convex. This type, which vibrates in an extensional (compressional) mode, is more difficult to excite into oscillation if the contour is flat or convex, that is, the resulting crystal units have low $Q$.

It therefore seemed logical that to obtain a good crystal by loading, the metal should be applied near the edges of the face rather than in the center, and the results justify this reasoning.

The plates were approximately $15 / 16$ in. square and had a contour such that the corners were 0.0002 in. thicker than the center. They were mounted in an adjustable air-gap holder, and the air gap was adjusted for maximum activity.

Because of the contour, all of the plates had fairly good characteristics before loading. One unit, $\mathrm{X}-2$, had an unloaded $Q$ of approximately 100,000 , but this was increased to approximately 262,000 by the additions of a load. The loaded area was a band $1 / 8$ in. wide along the edges, and the material was Wood's metal (fig. 9). Table 1 shows the resulting improvement in this unit. The small changes in $C_{0}$ are the result of varying the air gap to keep the activity at a maximum.

TABLE 1

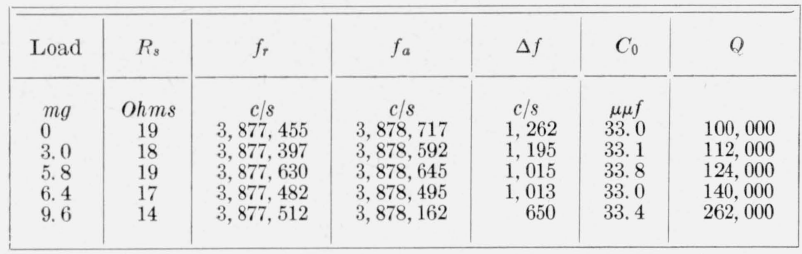

Slightly increasing the area by widening the load had the same effect as increasing the area of the load in the case of thickness-shear-mode plates. It resulted in an increase in $\Delta f$ but had no predictable effect on $R_{s}$.

\section{Summary}

The effects of loading vary not only with the type and condition of the oscillator plate but also with the size, position, and mass of the load. The first effect, observed in heavily loaded flat thickness-shear-mode plates, is probably due to the establishment of a large resonance frequency differential between the center and edges, causing the lower frequency vibrations originating in the central regions to be almost completely attenuated (fig. 10). Because the secondary modes coupled to the fundamental are confined to the central region and are effectively reduced in amplitude, the peripheral area of the plate is unable to oscillate and remains relatively inert. This concentration of activity in the center isolates the vibrating quartz and the mount and prevents reflections of energy from the crystal edges.

The effect of loading observable in concave tbickness-shear-mode plates and $\mathrm{X}$-cut plates operating in an extensional mode parallel to the thickness appears also to be one primarily of dimensioning certain areas by loading. The load here serves the purpose of slightly changing the frequency of certain regions of the plate so that the distribution of like frequency areas over the plate as a whole is more favorable for oscillation. In concave plates this is accomplished by the addition of the load to the central regions. It appears that thickness-shearmode crystals of this contour vibrate at a frequency above the optimum frequency of the plate as a whole because the central controlling area is the thinnest. The loading of the central area makes its frequency slightly lower, thereby establishing conditions more favorable for the plate as a whole. Flat or convex X-cut plates vibrating in extensional mode in the thickness direction are improved by the application of the load to the peripheral areas of the $\mathrm{X}$ faces. This improvement is possibly due to the surrounding quartz having a higher natural frequency than that of the central areas of the plate. Loading the outer areas tends to correct this condition by reducing the frequency of the higher frequency peripheral areas. This loading may be accomplished by applying a foreign material to the outer portions of the plate, as illustrated in figure 9 , or by hollow grinding the edges, as shown in figure 11 . In the latter case, the overhanging quartz may be thought of as constituting the load. This type of loading is more effective with plates of relatively low frequency. 


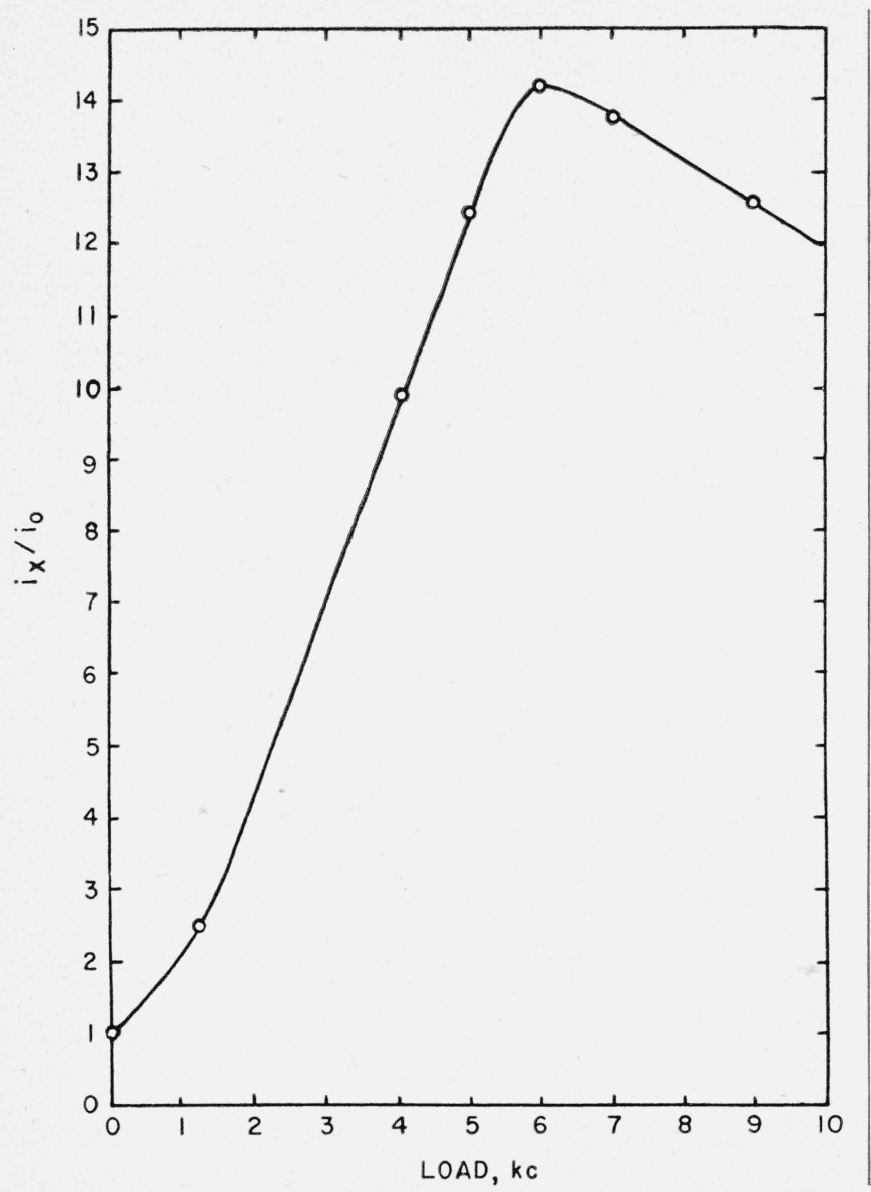

FIGURE 1. Effect of load on the activity (rectified grid current) of a concave BT-cut oscillator plate.

$i_{0}$ is the average activity with no load, and $i_{x}$ is the average activity with $x-\mathrm{kc}$ load.

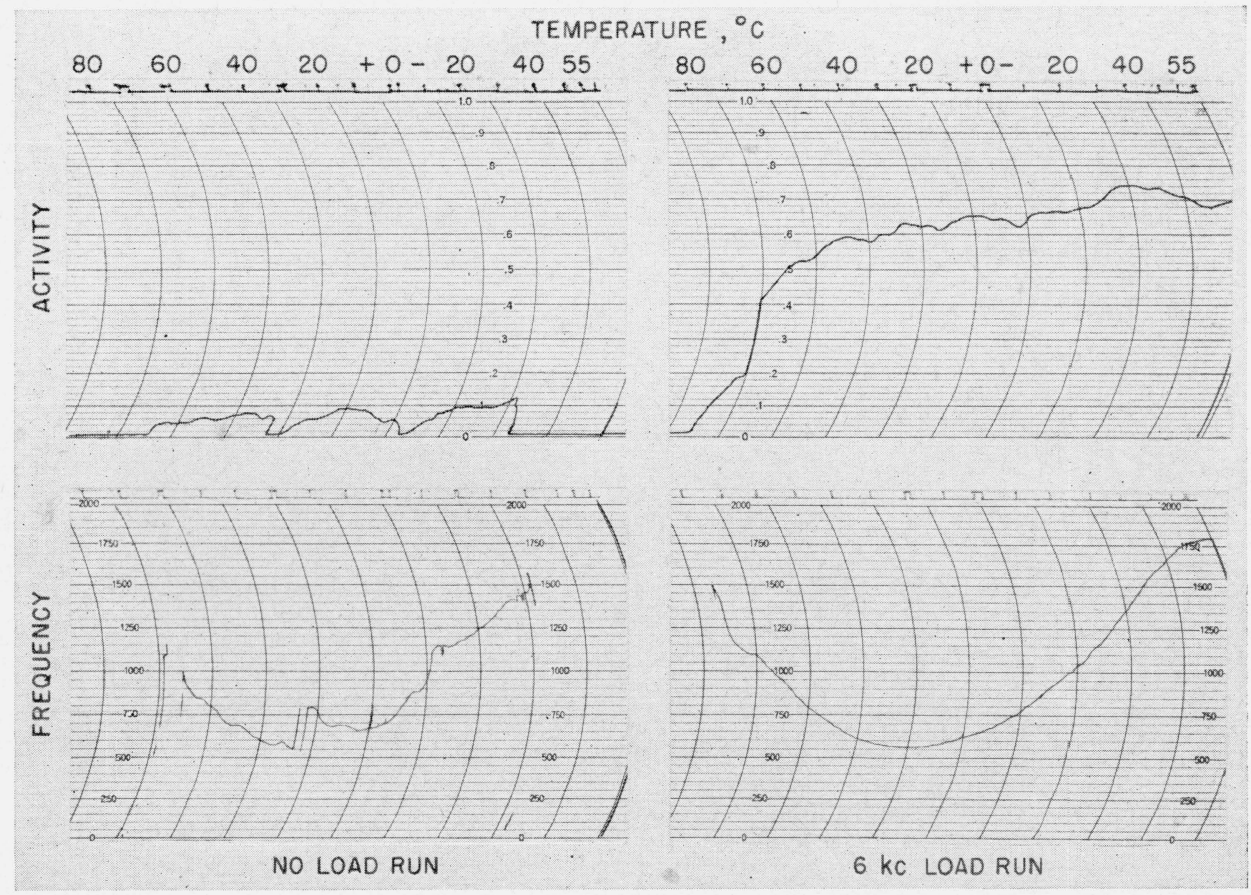

FIGURE 2. Improvement of a concave BT-cut oscillator plate $C C-8$ as a result of loading. The dip near $70^{\circ}$ in the loaded run is due to the melting of the Wood's metal. 


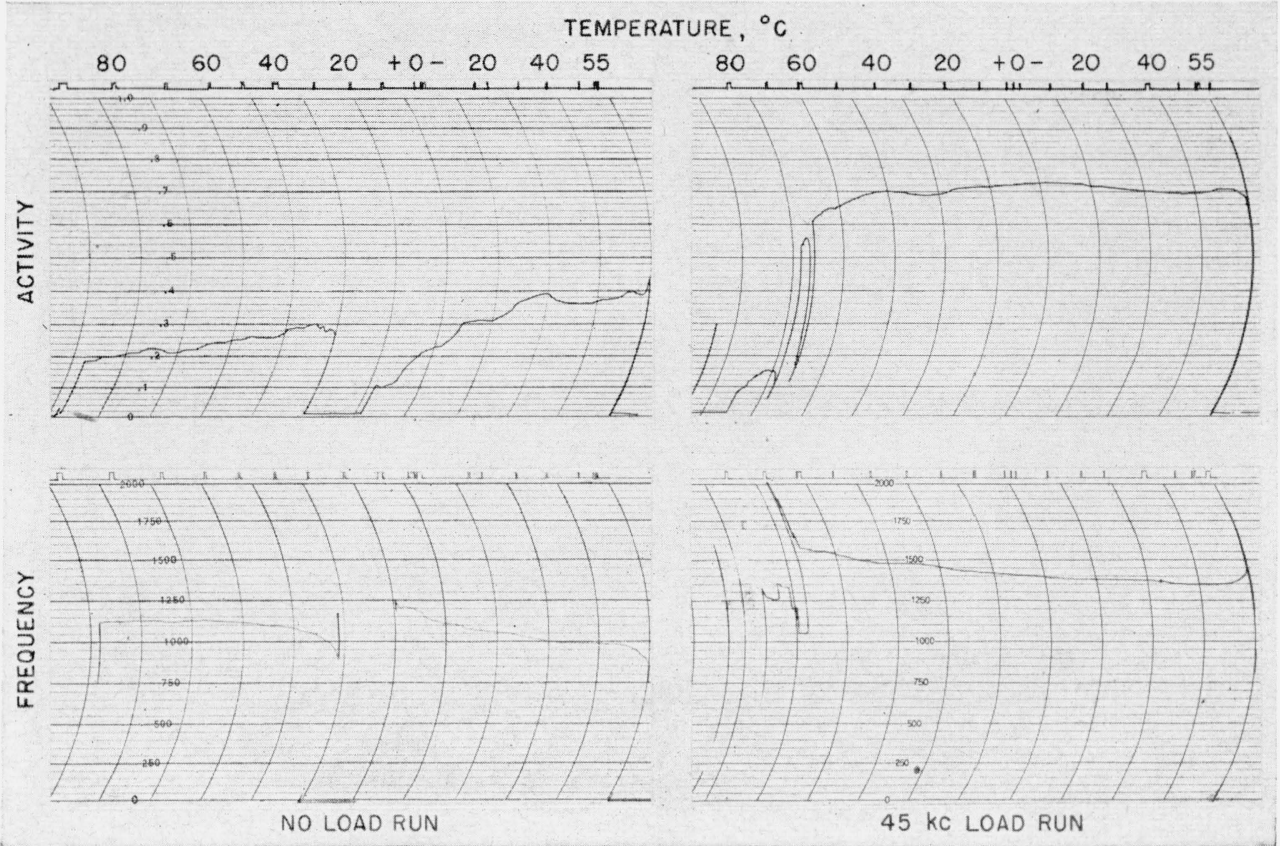

Figure 3. Improvement of a flat AT-cut oscillator plate AT-2 as a result of loading. The dip near $70^{\circ}$ in the loaded run is due to the melting of the Wood's metal.

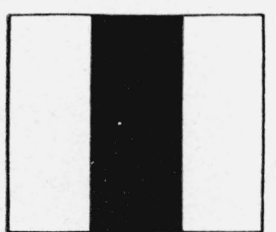

WIDE - BAND

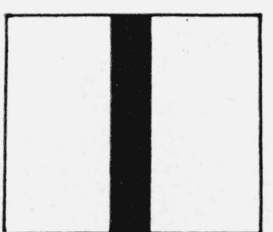

NARROW-BAND

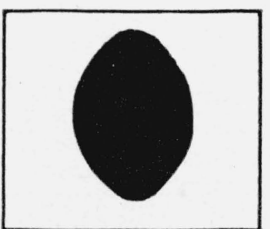

ELLIPTICAL

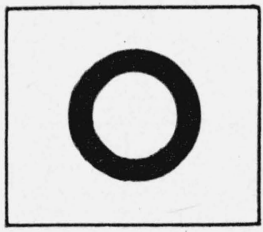

RING

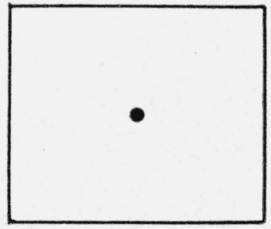

PIN-HEAD

FIGURE 4. Load patterns used in experiments.

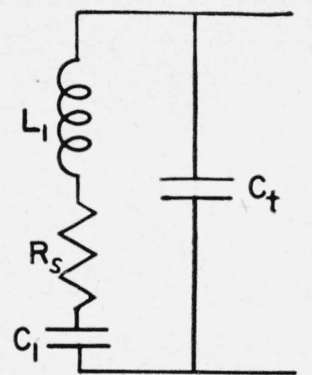

FIGURE 5. Electrical equivalent circuit of a quartz-crystal oscillator plate.

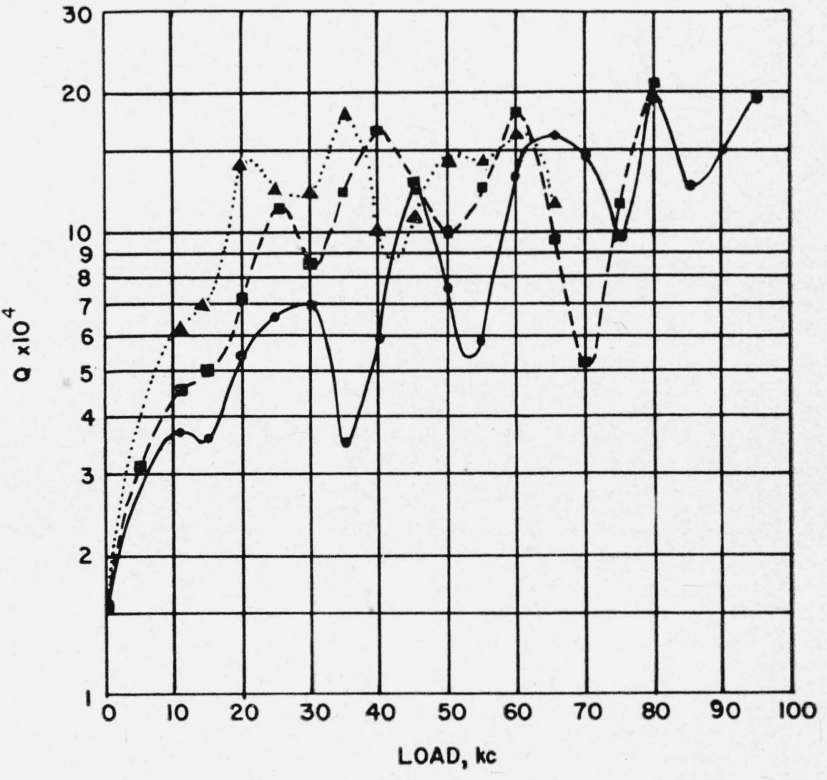

Figure 6. Improvement of $Q$ by loading for a flat $A T$-cut oscillator plate.

Load 1/3 length of $\mathrm{X}$ dimension.

Load $1 / 2$ length of $X$ dimension.

Width of all loads three-tenths width of oscillator plate. 


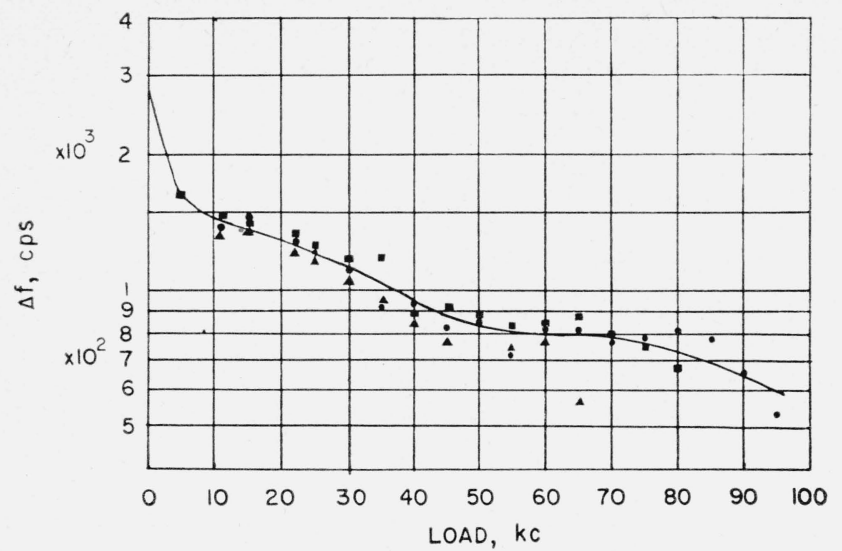

Figure 7. Decrease in $\Delta f$ as a result of loading.

A. Load $1 / 3$ length of $\mathrm{X}$ dimension.

Load $1 / 2$ length of $\mathrm{X}$ dimension.

, Load $3 / 4$ length of $\mathrm{X}$ dimension.

Width of all loads three-tenths,width of oscillator plate

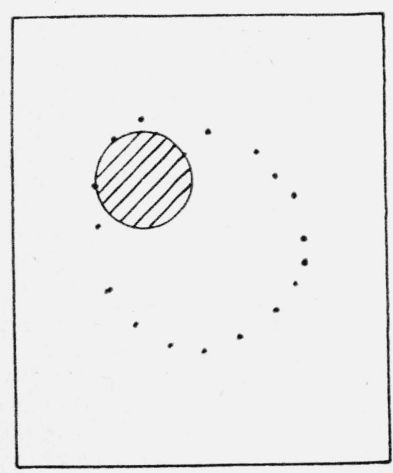

A

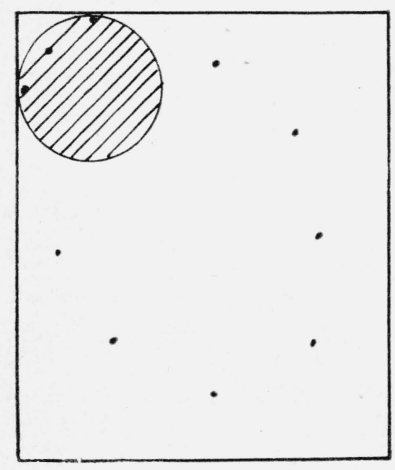

C

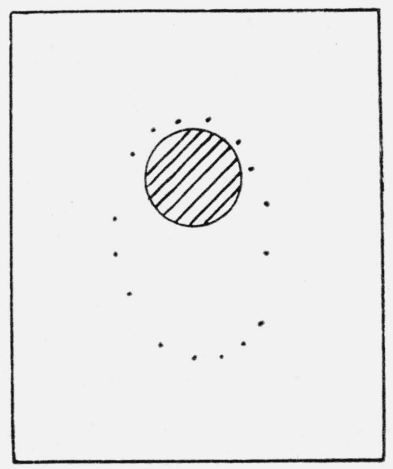

B

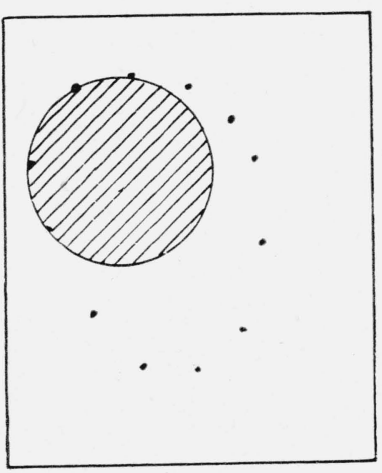

D
Figure 8. Active area of crystal plates with off-centered electrodes.

A, 1/8-in. electrode near to center in line with corner; B, 1/8-in. electrode tangent to center in middle of short dimension; C, 3/16-in. electrode in corner; D, $1 / 4$-in. electrode tangent to center in line with the corner.

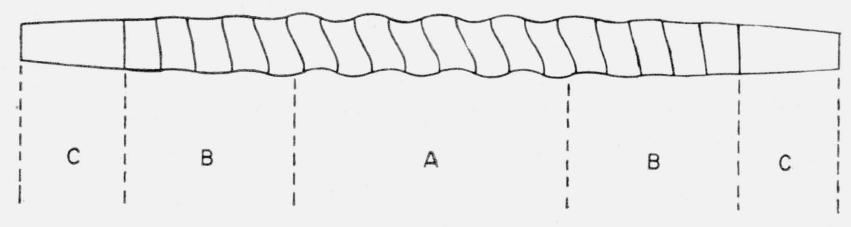

FIGURE 10. Idealized drawing of the cross section of a convex thickness-shear plate illustrating the damping effect of contouring.

Region A, active central area; B, damping area; C, inert quartz.

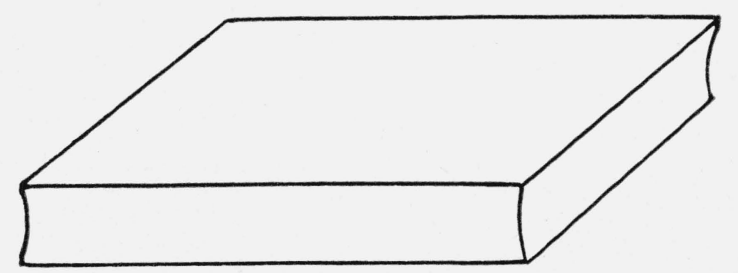

Figure 11. X-cut plate with hollow-ground edges.

Washington, June 17, 1952. 